\title{
Análise da Experiência do Usuário (UX) de Narrativa Transmídia através de Mouse-Tracking
}

\author{
User Experience (UX) Analysis of a Transmedia Narrative Using Mouse-Tracking
}

\author{
Rita de Cássia Romeiro Paulino ${ }^{\mathrm{ai}}$ \\ ORCID: https://orcid.org/0000-0002-3020-7091
}

Marcos César da Rocha Seruffo bii

ORCID: http://orcid.org/0000-0002-8106-0560

Marina Lisboa Empinotti $i^{\mathrm{ciii}}$

ORCID: http://orcid.org/0000-0003-3697-9962

Kennedy Edson Silva de Souza ${ }^{\mathrm{div}}$

ORCID: https://orcid.org/0000-0003-0056-2436

Ana Carla Pimenta
ORCID: https://orcid.org/0000-0002-2428-2941

Recebido em: 24 /05/2021. Aprovado em: 13/10/2021.

Resumo

Este artigo objetiva identificar as interações no site transmídia "De barrio somos" utilizando um sistema quantitativo de rastreamento de interações intitulado Artificial Intelligence and Mouse Tracking-Based user Experience Evaluation Tool (AIMT-UXT). Foram mapeadas ações nas categorias clique, espera, rolagem e movimentação do mouse para avaliar a experiência do usuário (UX). Recorremos à entrevista estruturada para obter dados para confrontar resultados analíticos das interações. $\mathrm{O}$ mapeamento foi feito com alunos de Jornalismo da Universidade Federal de Santa Catarina, que navegaram no site e participaram da entrevista. Como resultado, observamos um maior interesse nos audiovisuais.

Palavras-Chave: Mouse-tracking. Transmídia, De barrio somos.
Abstract

This paper aims to identify interactions on the De barrio somos transmedia website using a quantitative interaction tracking system entitled Artificial Intelligence and Mouse Tracking-Based user Experience Evaluation Tool (AIMT-UXT). Actions were mapped in the click, wait, scroll and mouse movement categories to assess the user experience (UX). We used the structured interview to obtain data to confront analytical results of the interactions. The mapping was done with Journalism students from the Federal University of Santa Catarina, who browsed the site and participated in the interview. As a result, we see a greater interest in audiovisual content.

Keywords: Mouse-tracking. Transmedia, De barrio somos.

\footnotetext{
a Universidade Federal de Santa Catarina. Santa Catarina/Brasil. E-mail: rcpauli@gmail.com

b Programa de Pós-Graduação em Estudos Antrópicos na Amazônia (PPGEAA) e Programa de Pós-Graduação em Engenharia Elétrica (PPGEE). Pará/Brasil. E-mail: marcos.seruffo@gmail.com

${ }^{c}$ Instituto Federal de Santa Catarina. Santa Catarina/Brasil. E-mail: marinaempinotti@gmail.com

${ }^{\mathrm{d}}$ Universidade Federal do Pará. Pará/Brasil. E-mail: kennedy.souza@castanhal.ufpa.br

${ }^{\text {e } U n i v e r s i d a d e ~ F e d e r a l ~ d e ~ S a n t a ~ C a t a r i n a . ~ S a n t a ~ C a t a r i n a / B r a s i l . ~ E-m a i l: ~ a n a c a r l a p i m e n t a 20 @ g m a i l . c o m ~}$
} 


\section{Introdução}

Entendemos a narrativa transmídia como um espaço que concatena elementos midiáticos que reunidos contam uma história, observando a singularidade de cada meio e contexto. Consideramos o desenvolvimento desse formato como um grande desafio para a área jornalística, por ser um produto que exige abordagem multidisciplinar, tornando possível a observação das impressões e percepções causadas no público por essa forma de consumo de mídia.

Segundo o padrão ISO 9241-210 (ISO, 2018), todas as impressões, percepções, emoções, crenças, preferências e comportamentos que ocorrem antes, durante e depois da utilização de um produto ou serviço compõem a Experiência de Usuário (UX), sendo, portanto, um aspecto cada vez mais crítico no desenvolvimento de novas formas de difusão de informação e conhecimento. A UX é um campo crescente, que vem se desenhando como de suma importância para qualificação e quantificação de imersão. Avaliar o design de um sistema considerando a UX pode levar a um maior índice de satisfação e, assim, a qualidade do design de UX pode ser avaliada usando ferramentas apropriadas para rastrear as atividades de cada usuário, conforme Cegan e Filip (2017).

Selecionamos para este estudo o site do projeto transmídia De barrio somos - Histórias de Clubes em $360^{\circ}$ para monitorar a Experiência do Usuário (UX) por utilizar recursos transmidiáticos e ter reconhecimento e premiações na área. De barrio somos apresenta características inovadoras (PIMENTA, 2019). Segundo a autora, foi concebido por uma equipe interdisciplinar que produziu uma série para TV e web, um livro de crônicas, conteúdos em $360^{\circ}$, um álbum de figurinhas com rastreadores de realidade aumentada, um jogo de tabuleiro, pequenas histórias para redes sociais e outros. Todos esses produtos foram reunidos e disponibilizados em um site que faz parte desse projeto transmidiático ao reunir os diversos elementos desenvolvidos.

\section{Objetivo}

Motivados pela questão de pesquisa "como observar a Experiência dos Usuários em sites com tantos elementos distintos?", recorremos à área da Computação para identificar 
técnicas que nos ajudassem a identificar o caminho percorrido pelo usuário nestas narrativas, suas ações e frustrações durante o processo interativo. Técnicas e métodos têm sido desenvolvidos com o intuito de melhorar a UX. O estudo do comportamento humano é de particular interesse no campo da Interação Humano-Computador (HCI), pois pode fornecer informações sobre o desempenho humano. A identificação de padrões de utilização do mouse, como movimentos aleatórios e retos, pairar o mouse etc., pode ser associada ao uso da facilidade percebida, utilidade percebida, autoeficácia, vontade de aprender ou percepção de risco (TZAFILKOU; PROTEGEROS, 2018).

Os testes de usabilidade são métodos utilizados para avaliação, centrados no usuário, e têm o objetivo de identificar como design, conteúdos e mídias podem ser melhorados durante o processo de desenvolvimento e avaliar a qualidade global da interface utilizando-se medidas de performance (PAULINO et al., 2018). Para identificar interações no site utilizamos um sistema de rastreamento de interações de usuários, a ferramenta intitulada Artificial Intelligence and Mouse Tracking-Based user Experience Evaluation Tool (AIMT-UXT), proposta por Souza et al. (2019).

Dados de monitoramento do comportamento do usuário obtidos pelo rastreamento do mouse podem ser utilizados para identificar problemas na navegação e interesses no conteúdo. A análise de dados advindos dos cliques, movimento do mouse, tempo de espera em conteúdos e rolamento nas seções do site são métricas com as quais podemos analisar a UX em qualquer sistema interativo, inclusive em um site, diagnosticando problemas e propondo melhorias para aumentar a experiência de uso (SOUZA et al., 2019).

\section{De barrio somos: contextualização do objeto empírico}

Em Rosário (Argentina), os clubes de bairro se configuram como comunidades ativas e de grande importância para a cidade. Nesse contexto é que o projeto De barrio somos se originou, divulgando a história e noticiando as atividades de cada clube. Mais do que clubes de futebol, os clubes de bairros de Rosário são espaços de encontro, servindo a cidade como uma esfera pública de valor para todos (PIMENTA, 2019).

O projeto promove um passeio transmidiático por histórias contadas pelos seus próprios personagens. Integrando várias mídias e produtos, faz um convite a navegar e explorar as 
culturas de bairro reveladas em $360^{\circ}$. O site se apresenta em um formato Longform (Figura 1) para contar uma história que se caracteriza por ter várias seções na orientação vertical.

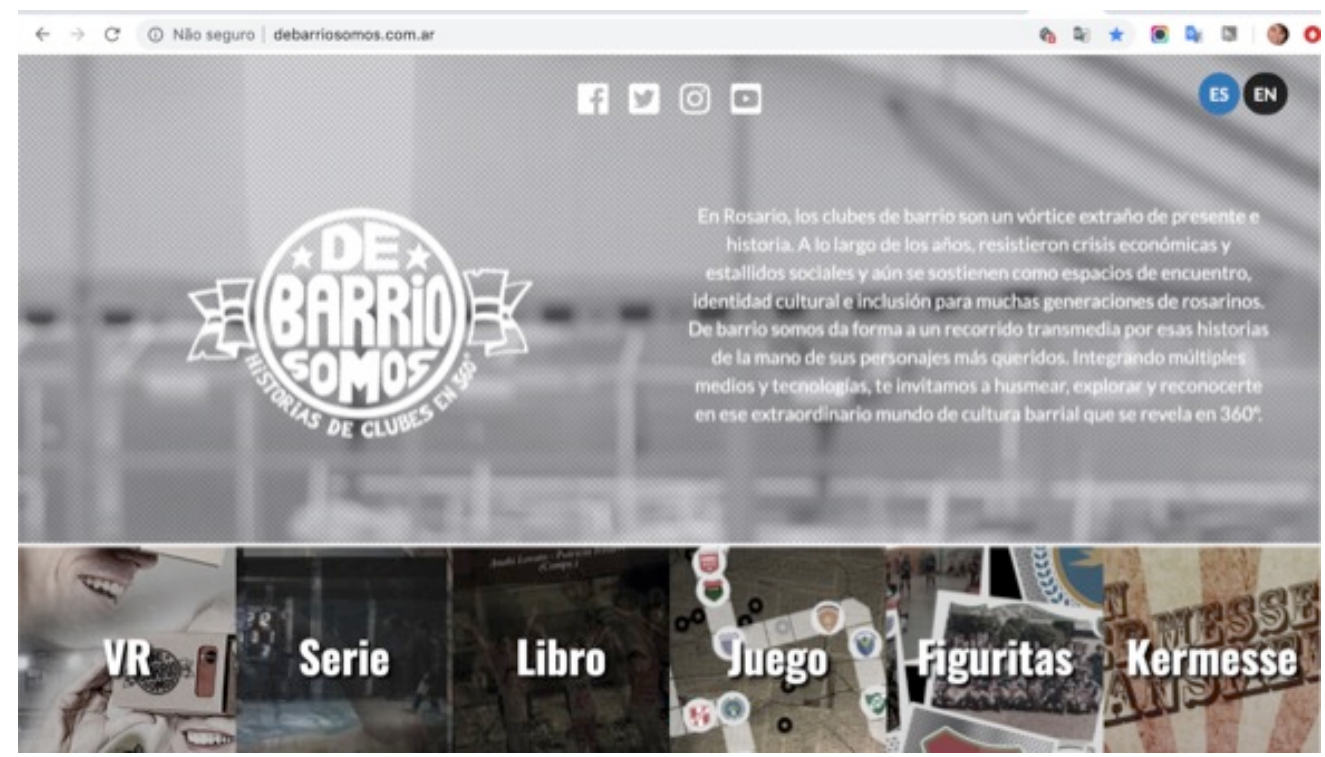

Figura 1 - Página principal do De barrio somos

Fonte: (DEBARRIOSOMOS, 2021)

De barrio somos contou com a participação de profissionais de diversas áreas. A equipe foi formada por jornalistas, comunicadores, produtores audiovisuais, designer gráfico e especialistas em tecnologia. O projeto foi financiado através de um edital público de captação financeira. Aqui é importante destacar que a produção foi desenvolvida pelo Departamento de Produções Transmídia da Universidade Nacional de Rosário (DCMteam), uma Instituição Federal Argentina e financiado pela Rede Nacional Audiovisual Universitária (RENAU) e pelo Programa Espacio Santafesino do Ministério de Inovação e Cultura de Santa Fé. Também contou com o apoio do Jornal La Capital, que divulgou uma série de artigos sobre o projeto (PIMENTA, 2019).

A equipe interdisciplinar produziu uma série para TV e web, um livro de crônicas, conteúdos em $360^{\circ}$, um álbum de figurinhas com rastreadores de realidade aumentada, um jogo de tabuleiro e pequenas histórias para redes sociais. Uma história transmídia desenrola-se através de múltiplas plataformas de mídia, com cada novo texto contribuindo de maneira distinta e valiosa para o todo. Na forma ideal de narrativa transmídia, cada meio faz o melhor - a fim de que uma história possa ser introduzida num filme, ser expandida pela televisão, 
romances e quadrinhos; seu universo possa ser explorado em games ou experimentado como atração de um parque de diversões (JENKINS, 2009).

Para o autor, cada acesso à franquia deve ser autônomo, de modo que não seja preciso assistir a um filme para gostar de um jogo ou vice-versa. O ponto de acesso à franquia pode, e deve, ser qualquer um dos itens componentes da narrativa. Ainda segundo Jenkins (2009), a primeira narrativa transmídia de que se tem conhecimento é a franquia A Bruxa de Blair (1999), pois, enquanto passava nos cinemas, já havia um site com informações e vídeos inéditos e opções de compras de mercadorias. "Interessante destacar que até hoje o site segue sendo atualizado, com novas experiências midiáticas como uma imersão em realidade virtual pela floresta onde se passa a história" (EMPINOTTI et al., 2018).

A análise do De barrio somos permite observar as semelhanças do projeto com os exemplos de transmídia apontados por autores como Jenkins (2009): diferentes mídias foram usadas de forma estratégica; o conjunto delas forma um todo mais completo, oferecendo uma experiência mais imersiva ao consumidor; o acesso à obra pode se dar por diversos pontos de entrada, sem prejuízo à compreensão, de forma que o usuário faz sua opção e trilha sua forma de consumo como preferir, assumindo papel decisivo sobre o que consome; em cada uma das mídias utilizadas, o conteúdo é moldado de forma a usá-la plenamente, apropriando-se de suas vantagens, em oposição ao que ocorre em estratégias crossmídia, em que se usam diversas plataformas simultaneamente, mas sem grandes alterações no conteúdo (BOUMANS, 2004).

Tais características nos levam a crer que lidamos com uma narrativa transmídia. Há ainda o fato de o site reunir todos os diversos conteúdos criados para a ação, incluindo versões digitais de jogos de tabuleiro e álbuns de figurinhas, indo ao encontro do que reconhecem Jenkins, Green e Ford (2015): os consumidores estão utilizando novas tecnologias para se envolver com conteúdos dos "velhos meios".

Apesar de muitos autores pensarem a transmídia sobretudo em situações de ficção, citando exemplos populares como as franquias Star Wars, Universo Marvel e Bruxa de Blair, narrativas transmídia já são consideradas em diversos contextos, como o aqui envolvido, da informação. Pesquisadores como Lähteenmäki (2021) expandem as reflexões de Jenkins (2009). Esse último afirma que a narrativa transmídia representa um processo em que elementos integrais de uma ficção são dispersos por vários canais de distribuição para criar uma experiência de entretenimento unificada e coordenada. 
Lähteenmäki (2021) traça um paralelo ao substituir o termo "ficção" por "história", resultando em: "A narrativa transmídia representa um processo onde elementos integrais da 'história' são dispersos sistematicamente por vários canais de entrega com o objetivo de criar uma experiência de entretenimento unificada e coordenada" (LÄHTEENMÄKI, 2021, p.8). Cabe ainda a ressalva de que "história" não deve ser confundida com "passado". Nessa situação, o uso do termo se refere a situações além das experiências de entretenimento, englobando também histórias orais, livros, artigos, documentários, exposições em museus e, como o caso em questão, produtos e serviços informacionais.

\section{Uso da tecnologia de Rastreamento (Tracking)}

A avaliação UX vem sendo realizada por vários trabalhos, que consideram diferentes técnicas e metodologias de rastreamento, como smart-wearables (PAL et al., 2019), jogos para celular (YU et al., 2018), expressão facial (MUNIM et al., 017) e rastreamento do olhar e do mouse (PENG; WEN-HUNG, 2016). Os métodos de avaliação de UX são partes importantes para garantir que o desenvolvimento de produtos ou serviços avance na direção certa e, como resultado, o efeito fique mais perto do atendimento das expectativas dos usuários, com UX positiva (RAJESHKUMAR; OMAR; MAHMUD, 2013).

No contexto das Narrativas Transmídias, entre as tecnologias de rastreamento, encontramos dois principais tipos que mapeiam a interação do usuário: o Mouse-tracking considera a análise dos movimentos do mouse em experimentos computadorizados e está se tornando cada vez mais popular na pesquisa psicológica. Os movimentos do mouse são tomados como um indicador de comprometimento ou conflito entre opções de escolha durante o processo de decisão (WULFF,, 2018). Já o Eye-tracking permite estabelecer elementos das páginas nos quais os usuários concentraram sua atenção e analisam se eles coincidem com os elementos relevantes da IA (LÓPEZ GIL et al., 2010). O rastreamento ocular permite a identificação de áreas de interesse relevantes em relação aos elementos das páginas da web necessárias para responder perguntas de um Teste de Estresse (RTE). Esse determina se os usuários do teste concentraram sua atenção, segundo López Gil et al. (2010).

Nesta pesquisa adotamos o Mouse-tracking. Apesar de existirem dezenas de ferramentas de análise visual, muitas monitoram apenas dados da sessão do usuário. A tecnologia adotada nesta pesquisa envolveu o desenvolvimento de uma solução própria com 
enfoque efetivo na experiência e no comportamento do usuário em um site. A AIMT-UXT dispõe dos recursos de mapas de calor e reprodução de interações baseados em rastreamento de mouse que podem ser facilmente exportados. (SOUZA et al., 2017).

Para Kieslich, et al. (2018), o Mouse-tracking inclui decisões sobre o procedimento inicial, as configurações de velocidade e aceleração do cursor e o modo de resposta (clique ou passe do mouse). Cada uma dessas opções visa garantir que todos os processos cognitivos relevantes para a decisão ocorrem enquanto o rastreamento está ativo (o que é, em muitos casos, o período entre o clique no botão iniciar e a seleção de uma das opções de resposta), para que o processo de interesse seja capturado nas trajetórias.

\section{Metodologia}

Diante de tantos produtos que foram criados para este site Transmídia, a nossa pergunta de pesquisa quer saber: quais dos produtos foram mais acessados pelos alunos de Jornalismo e o porquê. O método de mouse-tracking sinaliza os locais mais acessados, sendo plotados a partir de uma análise quantitativa, mas pretendemos, com a aplicação dos questionários, entender o porquê dos caminhos percorridos no site, para agregar aspectos de análise qualitativa.

Consideramos esta pesquisa social como experimental e aplicada, sendo previsto o emprego imediato numa realidade circunstancial para geração de novos conhecimentos (GIL, 1999). Para tanto, utilizamos uma abordagem exploratória, motivada por problemas definidos que podem gerar hipóteses pesquisáveis para estudos posteriores. Por ser uma pesquisa bastante específica, podemos afirmar que assume a forma exploratória e de um estudo de caso.

Em uma análise na Base de Periódico da Capes, usamos os termos relacionados às tecnologias de rastreamento Eye-tracking e Mouse-tracking, retornando 110.883 publicações para o primeiro e 63.105 publicações para o segundo. As áreas variaram entre: Humanities, Medicine, Psychology, Computer Science, Education, Visual Perception, dentre outras (Figuras 2 e 3). Como o nosso foco é o Mouse-tracking, refinamos a busca para encontrar registros que tivessem relação com a Comunicação e o Jornalismo.

Encontramos 301 trabalhos com os seguintes termos: (mouse AND tracking) AND TITLE-ABS-KEY (communication). Mesmo assim, refinamos ainda mais para identificar Mouse Tracking no universo dos sites, (TITLE-ABS-KEY (mouse AND tracking) AND 
TITLE-ABS-KEY (communication) AND TITLE-ABS-KEY (sites) e encontramos 18 documentos, mas somente um tangenciou a área que nos interessava, os Anais da $15^{\mathrm{a}}$ Conferência Internacional sobre Interação Humano-Computador, HCI International 2013.

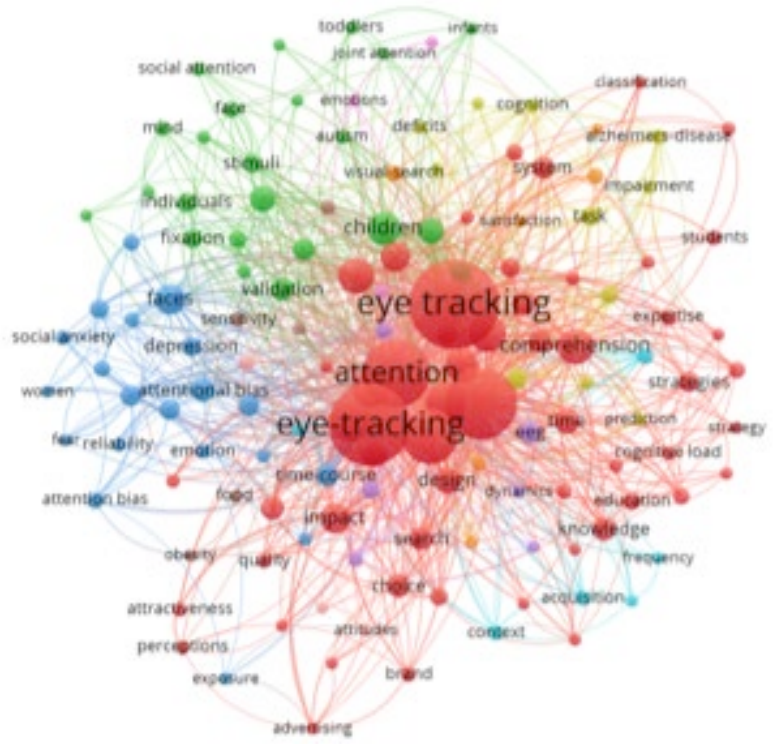

Figura 2 - Rede dos termos mais recorrentes na busca "Eye Tracking" na base do Scopus (Elsevier).

Fonte: Autores no VOSviewer. 


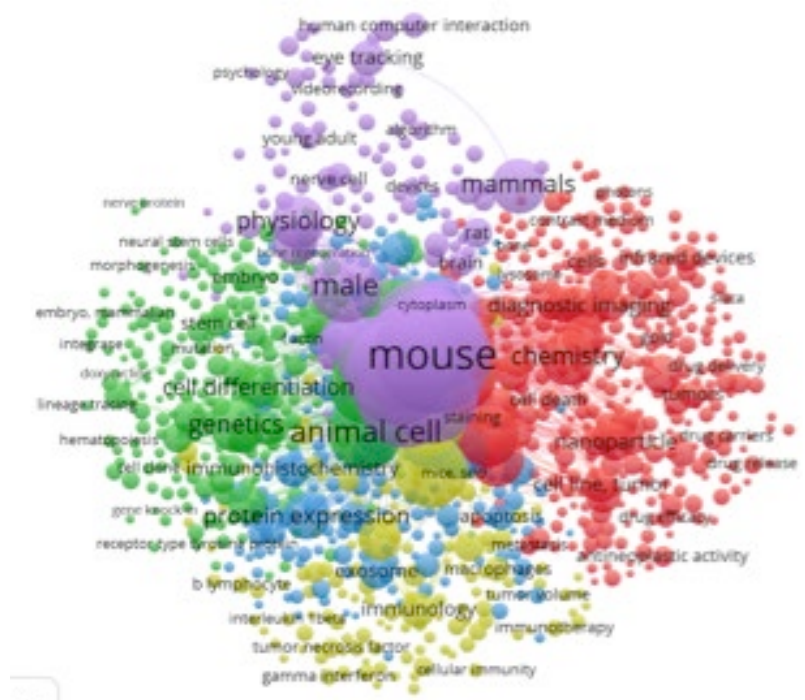

Figura 3 - Rede dos termos mais recorrentes na busca "Mouse Tracking" na base do Scopus (Elsevier). Fonte: Autores no VOSviewer.

Podemos considerar, portanto, que a área do Jornalismo e da Comunicação não se utiliza dessas técnicas para avaliar a interação em sites. Tal constatação corrobora o que afirmam Paulino et al. (2018): “a concepção e o desenvolvimento de softwares na área jornalística sempre foi uma caixa preta para os profissionais da área" (p. 40). É, assim, uma técnica amplamente utilizada e com experimentos documentados, mas pouco utilizada pela área da Comunicação para verificar a Experiência do Usuário (UX) em ambientes digitais.

Além da aplicação AIMT-UXT (análise quantitativa), recorremos à técnica da entrevista estruturada (análise qualitativa) com o objetivo de obter dados que comprovem ou não os resultados analíticos da interação dos usuários no site. O mapeamento foi feito com 13 alunos do curso de Jornalismo da Universidade Federal de Santa Catarina (UFSC) que navegaram no site De barrio somos e responderam a uma pergunta aberta para verificar a experiência de navegação indicando pontos positivos e negativos. Nos estudos de rastreamento de mouse, os movimentos do cursor dos participantes são registrados conforme os alunos escolhem entre diferentes opções representadas como botões na tela do computador. Assim, o rastreamento do mouse visa medir o grau de conflito entre as alternativas e o desenvolvimento temporal de sua resolução. 


\section{Tecnologia adotada - AIMT-UXT}

Analisar a interação do usuário em sistemas web nos permite aproximar a compreensão do grau de satisfação do usuário sobre o site. Neste artigo, um método sistemático de avaliar a UX usando métricas obtidas do rastreamento do mouse em combinação com medidas qualitativas será usado para mapear e entender as interações. Diante disso, Scores de avaliação e parâmetros de desempenho dos usuários serão identificados e mapeados.

A AIMT-UXT foi desenvolvida por Souza (2018) para coletar dados de interações do usuário relativos à utilização do mouse, podendo compor representações visuais possíveis de serem analisadas. A ferramenta disponibiliza três formas de visualização: reprodução de sessão, mapa de calor e tabulação. Os resultados obtidos servem de parâmetro para quantificar a UX que, comparada aos relatos do questionário aplicado (qualitativamente), revela-nos como foi essa interatividade e percepções de satisfação ou não. A ferramenta permite ainda a utilização de técnicas de inteligência artificial para mensurar a UX a partir de parâmetros de desempenho de usuários, entretanto, para esse estudo, essa função não foi necessária.

O rastreamento das interações no site, feitas pelos alunos, está presente na Tabela 1, que apresenta como resultado todas as interações dos usuários para cada objeto do site (objetos em HTML), descrito em uma tabela no formato .csv. A partir disso, foi possível fazer uma avaliação da ação tomada pelo usuário e validar as imagens apresentadas a seguir. As métricas analisadas são divididas em quatro tipos de atributos: Clique, Movimentação, Espera e Rolagem.

Tabela 1 - Tabulação contendo o mapeamento da interação coletada pela ferramenta AIMTUXT como resultado da navegação dos alunos no site De barrio somos.

\begin{tabular}{|c|c|c|c|c|c|}
\hline clicks & & & move & & \\
\hline qt & object & url & qt & object & url \\
\hline 48 & | img-fluid & debarriosomos.com. & 3304 & | img-fluid & debarriosomos.com. \\
\hline 7 & vid02 | img-fluid & debarriosomos.com. & 640 & | f1club2 f1cc w-100 & debarriosomos.com \\
\hline 6 & I fas fa-street-view fa- & - debarriosomos.com.i & 638 & | card-img-top & debarriosomos.com. \\
\hline 5 & jdescargar | nav-link & debarriosomos.com & 472 & | f1club5 f1cc w-100 & debarriosomos.com. \\
\hline 4 & vid03 | img-fluid & debarriosomos.com. & 398 & I f1club3 f1cc w-100 & debarriosomos.com. \\
\hline 4 & vid04 | img-fluid & debarriosomos.com. & 388 & frase2 | text-left & debarriosomos.com. \\
\hline 4 & jjuego | nav-link btju & debarriosomos.com. & 381 & | jumbotron bloque $c$ & debarriosomos.com. \\
\hline 4 & | f1club6 f1cc w-100 & debarriosomos.com. & 376 & | f1club6 f1cc w-100 & debarriosomos.com. \\
\hline 3 & vid07 | img-fluid & debarriosomos.com. & 360 & tv | jumbotron serie & debarriosomos.com. \\
\hline ? & I m19 An? & dahominammannm & s,o & 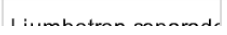 & Jahominommonsmm \\
\hline
\end{tabular}

Fonte: Autores. 


\section{Análise da aplicação dos testes com a Ferramenta AIMT-UXT}

Essa experiência foi realizada na disciplina Jornalismo On-line e Narrativas Digitais do Curso de Jornalismo da Universidade Federal de Santa Catarina em parceria com o Programa de Pós-Graduação Estudos Antrópicos na Amazônia - PPGEAA UFPA, na aplicação da tecnologia AIMT-UXT, desenvolvida por Souza (2018). Na primeira fase da pesquisa, os alunos tiveram uma breve explicação sobre o conceito e especificidades de um site transmídia. Após a apresentação, foram convidados a navegar pelo site De barrio somos em computadores tipo desktop que já estavam previamente relacionados com a Ferramenta AIMT-UXT instalada como uma extensão do navegador Google Chrome.

O usuário não percebe que a aplicação está sendo executada e utiliza normalmente o acesso ao site monitorado. Os dados coletados pela ferramenta são classificados nas quatro categorias previamente mencionadas, caracterizadas da seguinte maneira:

- Clique - refere-se ao clique do mouse na página e em determinado elemento html;

- Espera - quando o usuário deixa o mouse em repouso por mais de 3 segundos, o que sugere uma leitura da página;

- Rolagem - quanto o usuário utiliza roda de rolagem do mouse, indicando que o usuário procura por mais conteúdo na página;

- Movimentação - quando o mouse se movimenta na tela, são capturados os eixos (x e y) do mouse.

Todas as quatro categorias foram consideradas como indicadores que representam a navegação e indícios de consumo de informações. Para ressaltar as zonas do site em que houve um registro maior de tempo investido, utilizamos os mapas de calor.

A Figura 4 apresenta o mapa de calor, resultado das diferentes interações na página inicial. Destacamos Clique e Movimentação: houve muito movimento do mouse e clique sobre determinado objeto do site. Essa página mostra uma coleção de prêmios que o projeto De barrio somos ganhou ao longo do tempo. A Figura 4 indica que os alunos leram a introdução e seguiram para os dois prêmios destacados na parte superior. O interesse diminui ao seguir para a seção inferior. 


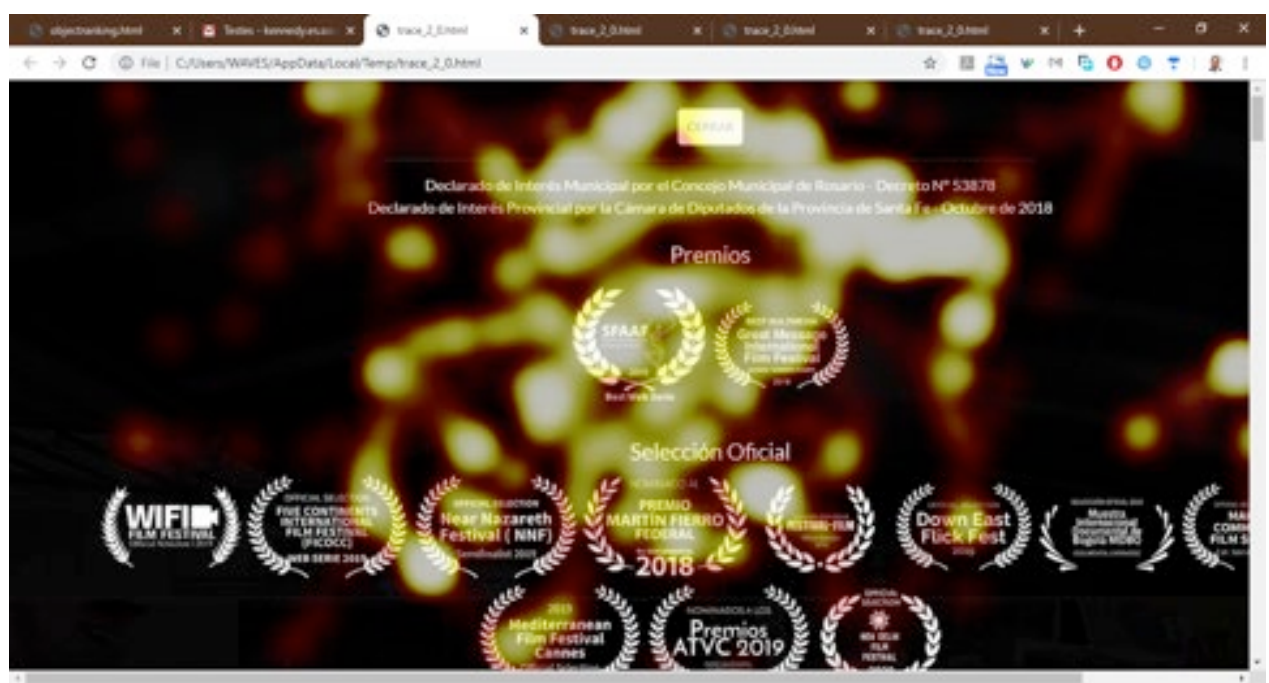

Figura 4 - Mapa de calor que representa as categorias Clique e Movimentação na página inicial. Fonte: Autores no site (DEBARRIOSOMOS, 2021)

A página "Serie", Figura 5, demonstra o interesse dos alunos em audiovisual. O sistema mostrou que o acesso a este tipo de conteúdo foi muito grande. Nessa página é apresentada uma Série documental de oito capítulos. Seus protagonistas nos convidam a visitar seus clubes e descobrir uma história polifônica com vários pontos em comum sobre paixão, amor pelas cores e construção coletiva de cada espaço de identidade do bairro.

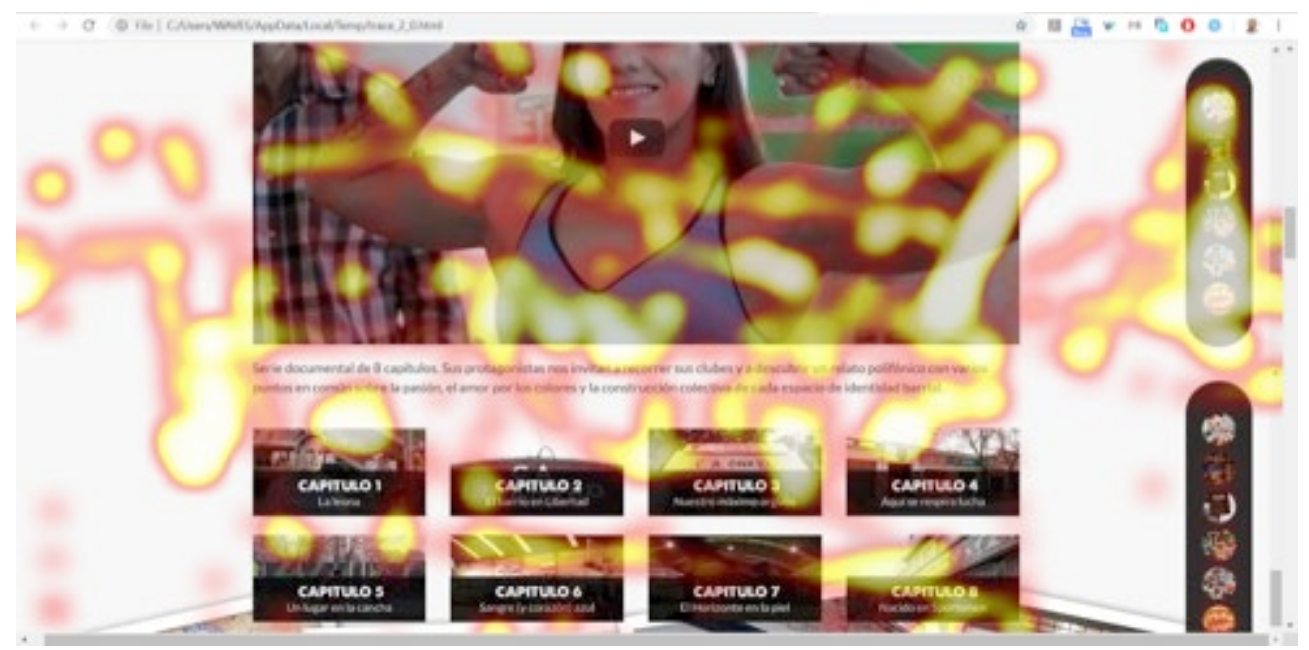

Figura 5 - Mapa de calor que representa as categorias Clique, Espera e Rolagem na página Séries.

Fonte: Autores no site (DEBARRIOSOMOS, 2021)

Na Figura 6, o Clique foi a categoria que mais se destacou no acesso aos vídeos em $360^{\circ}$. Essa constatação vai ao encontro do que foi relatado pelos alunos no preenchimento da 
questão aberta realizada após navegação. $\mathrm{O}$ vídeo $360^{\circ}$ foi o produto mais comentado e preferido pelos alunos. Os vídeos mais acessados foram "02. Tiro Suizo" e "05. Desportivo ligaUnión Central". O Tiro Suizo dá o nome a um bairro inteiro, no sul da cidade. Criado em 1889, suas cores (azul claro, branco e vermelho) unificam as bandeiras da Argentina e da Suíça. O time Deportivo Unión Central, popularmente conhecido como "La Carpita", o DUC foi fundado em 1946. Está localizado no coração do bairro Industrial.

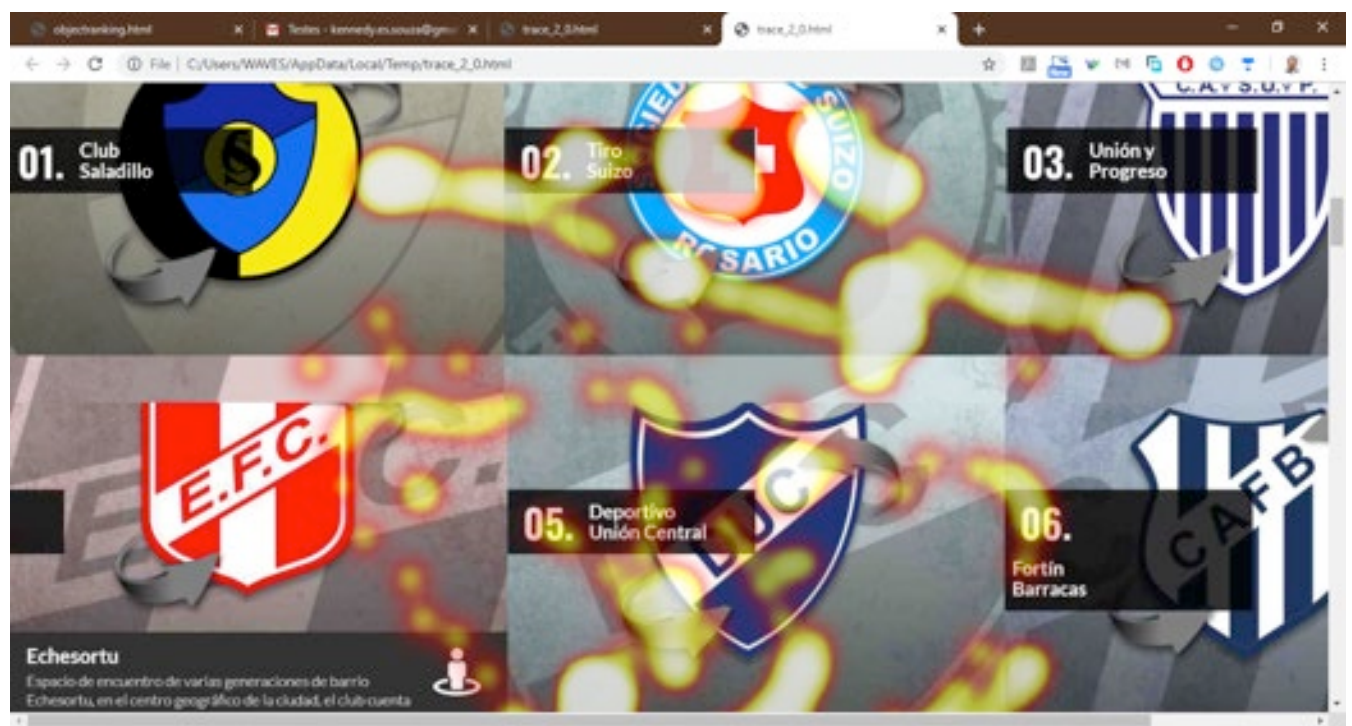

Figura 6 - Zonas de calor indica clube mais acessado entre as opções disponíveis na categoria Clique.

Fonte: Autores no site (DEBARRIOSOMOS, 2021)

Os objetos que concentraram mais Rolagens foram os identificados na página El diario La Capital (Figura 7). O site tem uma característica de Longform, então apresenta ações de rolagem de página na vertical. No entanto, o que chamou a atenção foi que nessa página de conteúdo não houve muita presença das demais categorias, o que sugere que o conteúdo textual naquele momento de navegação não cativou os alunos. O jornal La Capital apoia e promove o desenvolvimento do projeto De barrio somos através da divulgação de artigos semanais sobre multimídia. Sua publicação teve início em 20 de setembro de 2018. 


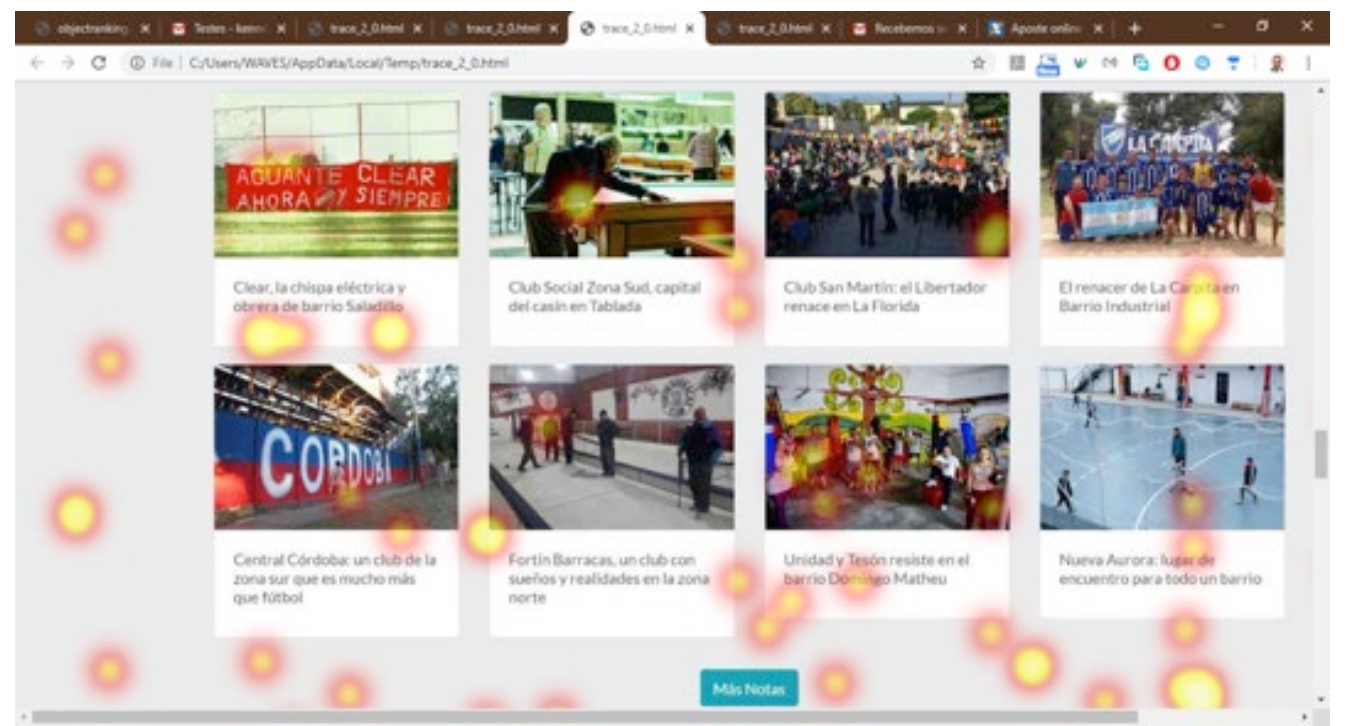

Figura 7 - Zonas de Calor em La Capital para a categoria Rolagem.

Fonte: Autores no site (DEBARRIOSOMOS, 2021)

Objetos que concentraram um nível alto de Rolagem e Movimentação estavam na página de Jogos (Figura 8). Os Jogos de tabuleiro são subprodutos muito acessados e, como existe a possibilidade de fazer download (baixar) no computador do usuário, notamos um acesso grande nessa página. Através dos Jogos de Tabuleiro, a história dos clubes do Rosário é contada. Nessa trilha, o desafio é descobrir fatos, campeonatos e heróis do bairro.

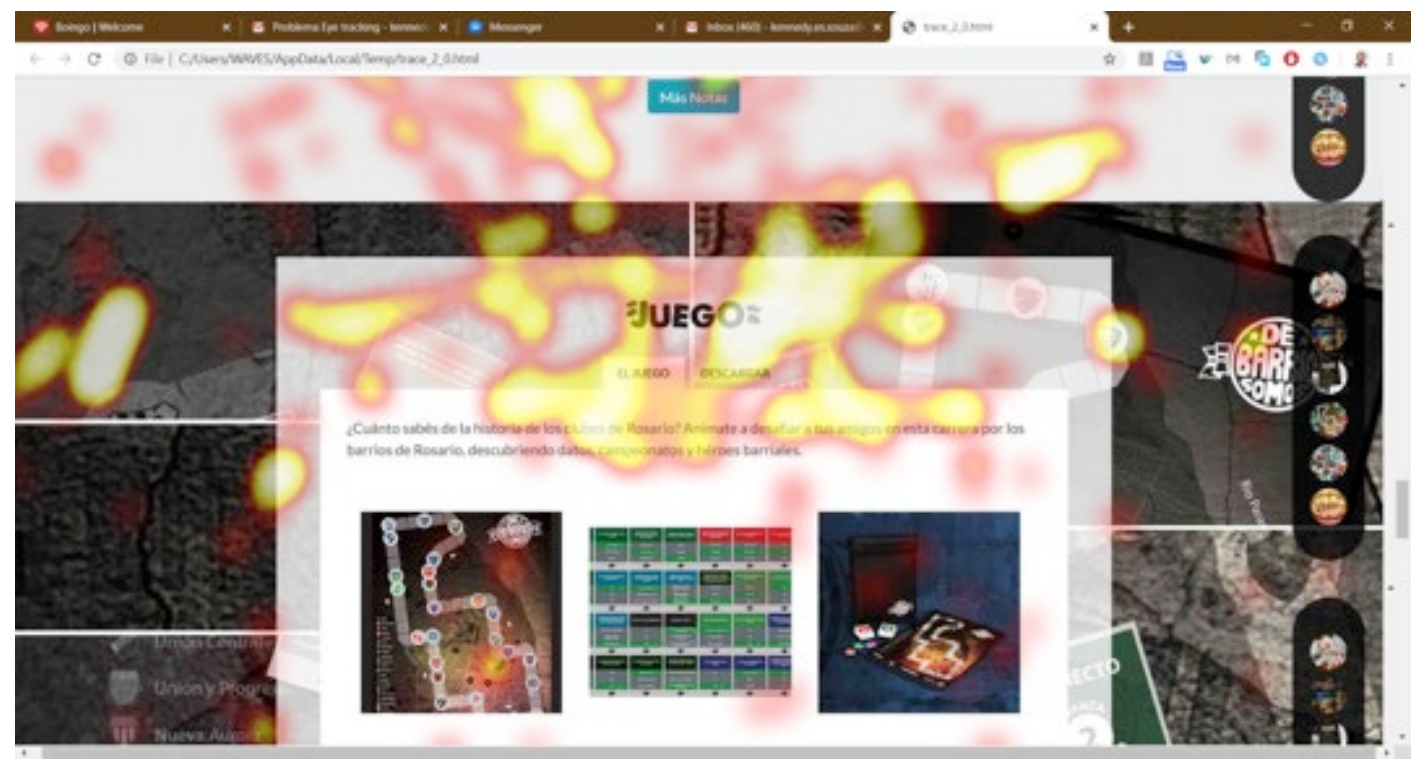

Figura 8 - Zonas de calor de Juegos para a categoria Movimentação

Fonte: Autores no site (DEBARRIOSOMOS, 2021) 
Novamente encontramos níveis altos de Rolagem e Movimentação na página Figuritas (Figura 9). O álbum de figurinhas é um dos subprodutos mais acessados e existe também a possibilidade de fazer download (baixar) no computador do usuário; notamos grande acesso nessa página. Na questão aberta, descrita no próximo capítulo, a possibilidade de fazer download dos produtos foi considerada como fator positivo. Os ídolos de cada clube, as copas e os campeonatos estão registrados nesse álbum de figurinhas.

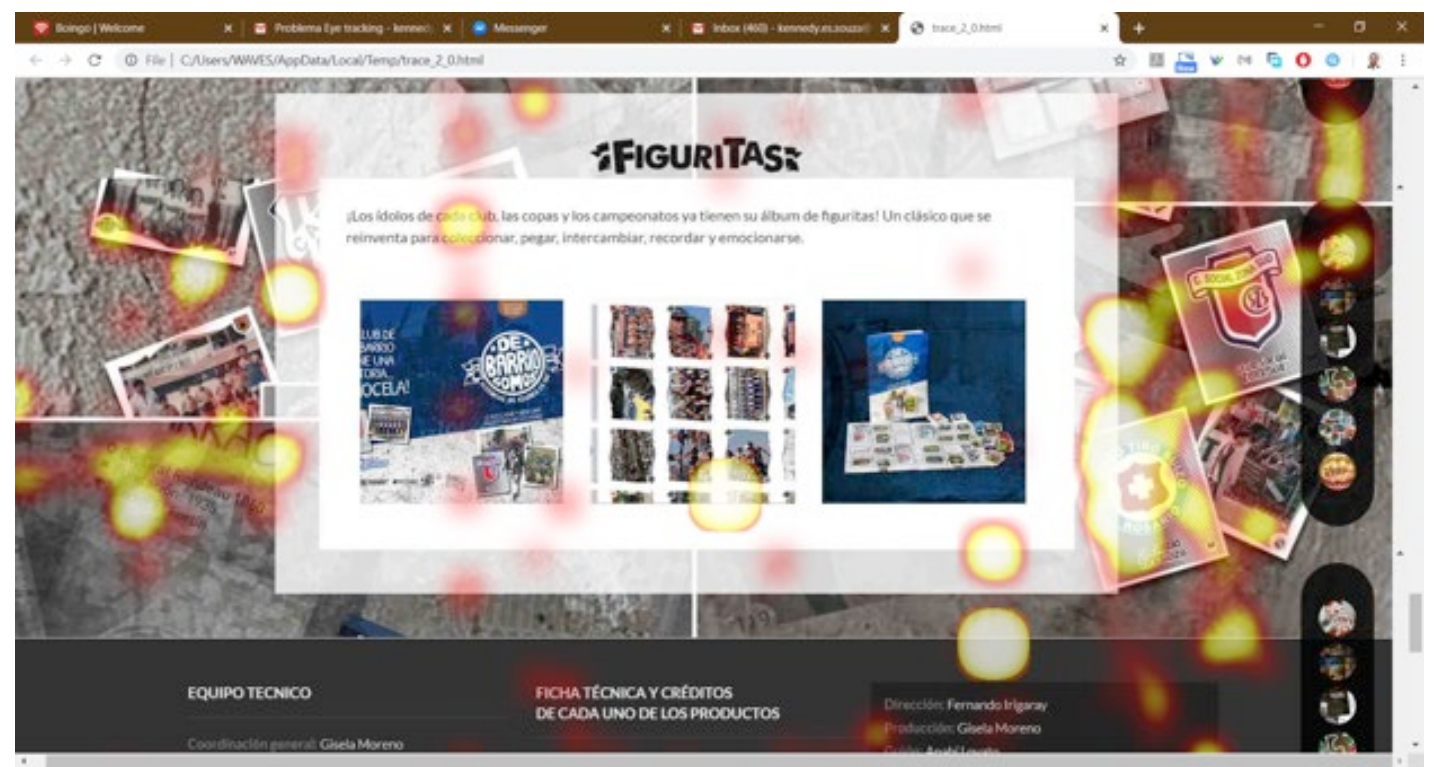

Figura 9 - Zonas de calor de Figuritas.

Fonte: Autores no site (DEBARRIOSOMOS, 2021)

\section{A perspectiva dos alunos}

Após a livre navegação no site, os 13 alunos do curso de Jornalismo que participaram da pesquisa foram convidados, de forma anônima, a responder a uma questão aberta sobre a experiência de navegar no site transmídia. O objetivo foi identificar os pontos positivos e negativos do site De barrio somos e obter informações que comprovem ou não os resultados analíticos da interação dos usuários no site.

Agrupamos os comentários nas categorias Aspectos de design e interatividade, Aspectos de Conteúdo e Produtos mais comentados (Quadro 1). A apresentação está subdividida, ainda, em Pontos Positivos e Pontos Negativos. 


\begin{tabular}{|c|c|}
\hline Pontos Positivos & Comentários \\
\hline $\begin{array}{l}\text { Aspectos de design } \\
\text { e interatividade }\end{array}$ & $\begin{array}{l}\text { "Gostei do site, achei muito bonito e fácil de navegar" } \\
\text { "O site é interessante, há vários tipos de mídia, o que agrega } \\
\text { muito." } \\
\text { "Gostei da diagramação da página principal e dos textos" } \\
\text { "A estética é bonita" } \\
\text { "O site é bonito" } \\
\text { "A diagramação é bonita, mas poderia ser mais básica, já que o } \\
\text { site tem muito conteúdo." } \\
\text { "Achei o site muito interessante e bonito." } \\
\text { "Traz muitos elementos de interatividade sem se tornar cansativo } \\
\text { e confuso." } \\
\text { "os menus estão claros e remetem aos conteúdos" } \\
\text { "...site é interessante e intuitivo" } \\
\text { "Os menus são claros e chamam o leitor a explorá-lo" } \\
\text { "O site é interessante, tem boa aparência" } \\
\text { "Mas o site é visualmente muito bonito" } \\
\text { "O site é muito bonito" } \\
\text { "O site é bem bonito, tem uma paleta de cores interessante" }\end{array}$ \\
\hline Aspectos de conteúdo & $\begin{array}{l}\text { "a proposta do site, de dar visibilidade para clubes de bairro, é } \\
\text { muito boa" } \\
\text { "O site é atrativo, principalmente para quem aprecie o conteúdo" } \\
\text { "Gostei muito dos conteúdos para baixar; constitui um conteúdo } \\
\text { extra divertido e com efeito de divulgação." } \\
\text { "A quermesse foi um evento bem planejado, também em relação } \\
\text { a divulgar o projeto." } \\
\text { "Passei boa parte do tempo lendo as notícias." } \\
\text { "A arquitetura das informações está ótima" } \\
\text { "No geral, é um site que desperta a curiosidade e nos faz querer } \\
\text { desbravar um assunto que, se tratado de outra forma, poderia não } \\
\text { interessar tanto" } \\
\text { "cada equipe tem um resumo da história e um botão que leva o } \\
\text { leitor a uma viagem pela sede do clube através do } 360^{\circ "} \\
\text { "No geral, o site é um deleite para os fãs de futebol amador } \\
\text { argentino" }\end{array}$ \\
\hline $\begin{array}{l}\text { Produtos mais } \\
\text { comentados }\end{array}$ & $\begin{array}{l}\text { "Curti as partes de vídeo e VR" } \\
\text { "Parei para assistir um vídeo que me chamou a atenção" } \\
\text { "A ferramenta do } 360 \text { foi bem interessante" } \\
\text { "Os vídeos em série foram uma das coisas que me chamaram } \\
\text { atenção" } \\
\text { "A visualização em } 360^{\circ} \text { me deixou muito envolvida, assim como } \\
\text { os minidocumentários" }\end{array}$ \\
\hline
\end{tabular}




\begin{tabular}{|c|c|}
\hline & $\begin{array}{l}\text { "o Ebook e a série de vídeos é um bom caminho para quem tiver } \\
\text { tempo" } \\
\text { "Adorei os vídeos na barra superior do site" } \\
\text { "Gostei bastante dos recursos de visão } 360^{\circ} \text {, etc." } \\
\text { "O elemento que mais me chamou a atenção foi o livro, } \\
\text { As fotos estavam boas também". }\end{array}$ \\
\hline Pontos Negativos & Comentários \\
\hline $\begin{array}{l}\text { Aspectos de design } \\
\text { e interatividade }\end{array}$ & $\begin{array}{l}\text { "Não achei boa a descrição do projeto em cima do vídeo em preto } \\
\text { e branco com a escrita branca, foi muito difícil de ler." } \\
\text { "A parte dos prêmios também não era muito legível } \\
\text { "...mas ter que scrollar tanto para chegar a uma informação que } \\
\text { não se tem certeza de onde se está é meio chato, e torna a } \\
\text { exploração do conteúdo lenta." } \\
\text { "Não entraria em um site cuja primeira página mostra os prêmios } \\
\text { que ele já ganhou, antes de qualquer outra coisa." } \\
\text { "...com os prêmios recebidos pelo site. Isso me deixou um tanto } \\
\text { confusa" } \\
\text { "Demorei pra me localizar pois tinha coisas demais." } \\
\text { "Em alguns momentos a legibilidade estava horrível." } \\
\text { "muitas ferramentas de interação" } \\
\text { "Acredito que deveriam ter feito um site mais minimalista" } \\
\text { "com a ressalva das fontes que destoam umas das outras, é algo } \\
\text { que pode ser mudado." } \\
\text { "Sobre sua funcionalidade, existem alguns links que não abrem } \\
\text { nada" } \\
\text { "Achei bem confuso, não curto longforms" } \\
\text { "Além de tudo não achei muito intuitivo e existem muitas coisas } \\
\text { escondidas que você precisa passar muito tempo para achar." } \\
\text { "O menu que "pipoca" no canto direito, não é muito funcional, } \\
\text { porque você tem que rolar a página para ele aparecer de novo." }\end{array}$ \\
\hline Aspectos de conteúdo & $\begin{array}{l}\text { "...mas sobrecarregado de informação. } \\
\text { Acho que seria mais interessante deixar evidente que são clubes } \\
\text { sociais." }\end{array}$ \\
\hline $\begin{array}{l}\text { Produtos mais } \\
\text { comentados }\end{array}$ & $\begin{array}{l}\text { "Demora para carregar" } \\
\text { "As logomarcas dos clubes que aparecem no início e interagem } \\
\text { com o mouse parecem botões/links sem ser". } \\
\text { "Os botões do site na parte } 360 \% \text { não são tão simples de } \\
\text { visualizar." }\end{array}$ \\
\hline
\end{tabular}

Quadro 1 - Análise Qualitativa do resultado de questão aberta aos usuários após navegação. Fonte: Autores. 
Podemos verificar que a técnica do mouse-tracking fornece indícios para identificar problemas que foram confirmados nos comentários dos usuários. Na página principal do site De barrio somos, que apresenta os prêmios do projeto, o sistema AIMT-UXT identificou muitas atividades da categoria Espera e Rolagem. Nessa página recebemos o maior número de comentários sobre o conteúdo exibido. É possível destacar alguns constantes no Quadro 1: "A parte dos prêmios também não era muito legível", "Em alguns momentos a legibilidade estava horrível." Isso indica que realmente os alunos dedicaram tempo de navegação a essa página, interessaram-se pelo conteúdo, mas tiveram dificuldades para a leitura.

Os comentários revelaram que a maior parte dos alunos gostou do Design e Interatividade e algumas páginas tiveram destaque, como a Série documental de 8 capítulos e o vídeo em $360^{\circ}$ que apresenta o time "Tiro Suizo". O objetivo social do site também foi reconhecido nos comentários dos alunos: "No geral, o site é um deleite para os fãs de futebol amador argentino". A ferramenta AIMT-UXT identificou muitos acessos nas páginas dos jogos e álbum de figurinhas, itens integrantes do caráter transmidiático do site.

\section{Considerações Finais}

Neste artigo foi utilizada a ferramenta AIMT-UXT, que utiliza a técnica de mousetracking para rastreio dos movimentos do mouse. Essa técnica foi aplicada no site De barrio somos (DEBARRIOSOMOS, 2021) , para verificar a navegação e interesses dos alunos nos produtos transmidiáticos. De acordo com os gráficos apresentados, que relatam os posicionamentos do mouse nas telas, podemos identificar as mais acessadas e os produtos de maior interesse pelos alunos.

Ter usado essas técnicas ajudaram a fazer uma avaliação mais criteriosa de interesses e problemas que foram diagnosticados a partir dos movimentos do mouse no site. Trata-se de uma ferramenta emergente que oferece uma janela acessível, rica em dados e em tempo real sobre como as pessoas categorizam e tomam decisões (STILLMAN, 2018).

A partir desses resultados, toda a movimentação do usuário na página é demonstrada em mapas de calor. Quando aplicamos instrumentos que identificam quantitativa e qualitativamente a interação e experiência do usuário (UX), podemos ter indícios mais concretos dos problemas que estão interferindo na qualidade da interação, ou dos aspectos em que o site foi mais eficiente. Como trabalhos futuros pretendemos investigar e aplicar o 
rastreamento do olhar (EyeTracking) para mapear a atenção dos usuários. Além disso, os dados obtidos serão tratados com modelos de inteligência computacional, na forma de mapas autoorganizáveis, com a finalidade de melhorar a clusterização das categorias mapeadas. Consideramos importante a utilização de novos métodos digitais emergentes, mesmo que não habituais na área da Comunicação, mas que abrem uma janela de colaboração entre as áreas e, principalmente, ajudam-nos na resolução de problemas.

\section{Referências}

BOUMANS, J. Crossmedia: e-content report 8. ACTeN - Anticipating Content Technology Needs, 2004.

CEGAN L.; FILIP P. Advanced web analytics tool for mouse tracking and real-time data processing. In: IEEE 14TH INTERNATIONAL SCIENTIFIC CONFERENCE ON INFORMATICS, 2017.

DEBARRIOSOMOS. Historias de Clubes en $\mathbf{3 6 0}^{\mathbf{0}}$. Disponível em: $<$ https://(DEBARRIOSOMOS, 2021)> Acesso em: 30 nov. 2021.

ISO. Iso 9241-11:2018 - ergonomics of human-system interaction - part 11: Usability: Definitions and concepts. Mar.[Online], 2018.Disponível em: https://www.iso.org/standard/63500.html Acesso em: 30 mar. 2021.

EMPINOTTI, M. L.; PAULINO, R. de C. R.; CAMINADA, T. de A. Estratégia Transmídia Em Plataformas Digitais: A Experiência Das Palavras Cruzadas Do The New York Times. Tropos: Comunicação, Sociedade e Cultura (ISSN: 2358-212X), [S. 1.], v. 7, n. 1, 2018. Disponível em: https://periodicos.ufac.br/index.php/tropos/article/view/1905 Acesso em: 30 mar. 2021.

JENKINS, H. Cultura da Convergência. 2. ed. São Paulo: Aleph, 2009

JENKINS, H; FORD, S; GREEN, J. Cultura da conexão: criando valor e significado por meio da mídia propagável. São Paulo: Aleph, 2015.

KIESLICH, P; HENNINGER, F; WULFF, D; HASLBECK, J; SCHULTEMECKLENBECK, M. Mouse-tracking: A practical guide to implementation and analysis, 2018. DOI 10.31234/osf.io/zuvqa.

LÄHTEENMÄKI, I. Transmedia history, Rethinking History, v. 25, n.3, p.281-306, 2021. DOI 10.1080/13642529.2021.1963597

LECKNER, S. Presentation factors affecting reading behaviour in readers of newspaper media: an eye-tracking perspective. Visual Communication, v. 11, n. 2, p. 163-184, 2012. DOI https://doi.org/10.1177/1470357211434029. 
LÓPEZ GIL, J; NAVARRO-MOLINA, C; GARCIA, R; ALEIXANDRE-BENAVENT, R. Analysis of website architecture through navigation stress tests, usability and eye tracking. The Information Professional, v. 19, n. 4, p. 359-367, 2010.

K. M. MUNIM, I. ISLAM, M. KHATUN, M. M. KARIM AND M. N. ISLAM, "Towards developing a tool for UX evaluation using facial expression," In: 3rd International Conference on Electrical Information and Communication Technology (EICT), 2017, pp. 1-6, doi: 10.1109/EICT.2017.8275227.

D. PAL, V. VANIJJA, C. ARPNIKANONDT, X. ZHANG AND B. PAPASRATORN, "A Quantitative Approach for Evaluating the Quality of Experience of Smart-Wearables From the Quality of Data and Quality of Information: An End User Perspective," in IEEE Access, vol. 7, pp. 64266-64278, 2019, doi: 10.1109/ACCESS.2019.2917061.

PAULINO, R; EMPINOTTI, M; JERONIMO, P. Experiência do Usuário (UX) em apps de conteúdo jornalístico. In: CANAVILHAS, J.; RODRIGUES, C.; GIACOMELLI, F. (Orgs.). Narrativas jornalísticas para dispositivos móveis, 1ed. Covilhã: LabCom - Universidade Beira Interior, v. 1, 2019, p. 33-56.

C. PENG AND W. LIAO, "Evaluation of Interactive Data Visualization Tools Based on Gaze and Mouse Tracking," 2016 IEEE International Symposium on Multimedia (ISM), 2016, pp. 431-434, doi: 10.1109/ISM.2016.0099.

PIMENTA, A. C. A transmidialidade no projeto De barrio somos. In: ANAIS DO $42^{\circ}$ CONGRESSO BRASILEIRO DE CIÊNCIAS DA COMUNICAÇÃO, 2019, Belém.

S. RAJESHKUMAR, R. OMAR AND M. MAHMUD, "Taxonomies of User Experience (UX) evaluation methods," In: International Conference on Research and Innovation in Information Systems (ICRIIS), 2013, pp. 533-538, doi: 10.1109/ICRIIS.2013.6716765.

SOUZA, K. Avaliação da experiência do usuário utilizando o rastreio de mouse e inteligência artificial. 2018. Trabalho de Conclusão de Curso (Bacharelado em Sistemas de Informação) -- Campus Universitário de Castanhal, Universidade Federal do Pará, Castanhal, 2018. Disponível em: https://bdm.ufpa.br/jspui/handle/prefix/2187. Acesso em: 30 mar. 2021.

SOUZA, K; SERUFFO, M; MELLO, H; SOUZA, D.; VELLASCO, M. User Experience Evaluation using Mouse Tracking and Artificial Intelligence. IEEE Access, v. 1, p. 1-10, 2019.

STILLMAN, P. How Mouse-tracking Can Advance Social Cognitive Theory. Trends in cognitive sciences (Regular ed.), v. 22, n. 6, p.:531 -543, 2018. ISSN 1364-6613

TZAFILKOU, K; NICOLAOS, P. Mouse Behavioral Patterns and Keystroke Dynamics in End-User Development: what can they tell us about users' behavioral attributes? Computers in Human Behavior. v. 83, p. 288-305, jun. 2018. DOI https://doi.org/10.1016/j.chb.2018.02.012 
YU, Q.; CHE, X.; MA, S.; PAN, S.; YANG, Y.; XING; W.; WANG, X. A hybrid user experience evaluation method for mobile games. In: Special Section On Big Data Learning and Discovery. IEEE Access, vol. 6, 2018, pp. 49 067-49 079.

\begin{abstract}
${ }^{\mathrm{i}}$ Pós-Doutora, ex-bolsista do CNPq no Social Media Lab na Universidade de Ryerson, Toronto - Ca em 2020. Concluiu o Doutorado no Programa de Pós-Graduação Engenharia e Gestão do Conhecimento, na área de Mídia e Conhecimento, pela Universidade Federal de Santa Catarina em 2011. Atua como Professora no Programa de Pós-Graduação em Jornalismo (PPGJOR), docente do curso de Jornalismo da Universidade Federal de Santa Catarina, nas disciplinas WebDesign e desenvolve pesquisa aplicada experimental nas áreas de Conteúdo interativo Multiplataforma, Aplicativos PWA (Progressive Web Apps), Análise de Redes Sociais e Jornalismo de Dados, tem experiência profissional como WebDesign Master. Atua também na área de Design e Comunicação, com ênfase em Comunicação Visual e Diagramação.
\end{abstract}

ii Realizou Pós-Doutorado na Pontifícia Universidade Católica do Rio de Janeiro, no Programa de Pósgraduação em Engenharia Elétrica (PPGEE - PUC-RJ - 2020) com bolsa Capes via projeto PROCAD Amazônia. Doutorado em Engenharia Elétrica, com ênfase em Computação Aplicada (PPGEE - UFPA - 2012). Mestrado em Ciências da Computação (PPGCC - UFPA - 2008). Especialização lato sensu em Suporte Técnico de Sistemas Computacionais pela Universidade Federal do Pará (UFPA - 2005). Graduação em Tecnologia em Processamento de Dados pelo Centro Universitário do Estado do Pará (CESUPA - 2004). Atua no Programa de Pós-graduação em Estudos Antrópicos na Amazônia (PPGEAA) e Programa de Pós-graduação em Engenharia Elétrica (PPGEE), além do curso de graduação em Engenharia da Computação. É Pesquisador do Laboratório de Pesquisa Operacional (LPO), coordenando e participa de atividades de ensino, pesquisa e extensão através de projetos nacionais e internacionais em áreas interdisciplinares, dentre estas: Análise de Redes Sociais, Tecnologias Sociais, Experiência do Usuário, Mineração de Dados e Informática na Educação. É membro da sociedade Brasileira de Computação.

iii Doutora em Estudos da Comunicação (FCT) na Universidade da Beira Interior, em Portugal. Jornalista e mestre em Jornalismo pela UFSC. Professora de Comunicação Visual do IFSC campus Palhoça Bilingue.

iv Bacharel em Sistemas de Informação pela Universidade Federal do Pará (2018), foi aluno de Iniciação Científica, e atualmente é Mestrando em Estudos Antrópicos na Amazônia, também pela UFPA/Castanhal. É bolsista de mestrado financiado pela Coordenação de Aperfeiçoamento de Pessoal de Nível Superior (CAPES), atuando nas áreas de Tecnologias Sociais, Experiência de usuário e usabilidade. É programador C\#, JavaScript e Lua, possuindo também experiência em C++, VB, Java e PHP, além de habilidades em UI design.

v Mestranda no Programa de Pós-Graduação em Jornalismo da Universidade Federal de Santa Catarina (Linha de pesquisa: Tecnologias, Linguagens e Inovação no Jornalismo). Graduada em Comunicação Social - Habilitação Jornalismo pelo Centro Universitário Estácio de Santa Catarina. Pesquisadora do Núcleo de Estudos e Produção em Hipermídia aplicados ao Jornalismo (Nephi-Jor), inserido no Grupo de Pesquisa Hipermídia e Linguagem CNPq. 